\title{
Low serum enterolactone concentration is associated with low colonic Lactobacillus-Enterococcus counts in men but is not affected by a synbiotic mixture in a randomised, placebo-controlled, double-blind, cross-over intervention study
}

\author{
Reetta Holma ${ }^{1 *}$, Riina A. Kekkonen ${ }^{2}$, Katja Hatakka ${ }^{2}$, Tuija Poussa ${ }^{3}$, Heikki Vapaatalo ${ }^{1}$, \\ Herman Adlercreutz $z^{4}$ and Riitta Korpela ${ }^{1}$ \\ ${ }^{1}$ Institute of Biomedicine, Pharmacology, PO Box 63, FIN-O0014 University of Helsinki, Finland \\ ${ }^{2}$ Valio Limited, RED, PO Box 30, FIN-O0039 Helsinki, Finland \\ ${ }^{3}$ STAT-Consulting, Vabverokatu 6, FIN-37130 Nokia, Finland \\ ${ }^{4}$ Institute for Preventive Medicine, Nutrition and Cancer, Folkhälsan Research Center, and Division of Clinical Chemistry, \\ Biomedicum, FIN-OOO14 University of Helsinki, Finland
}

(Submitted 9 November 2012 - Final revision received 11 April 2013 - Accepted 25 June 2013 - First published online 7 August 2013)

\section{Abstract}

The aims of the present study were to assess the possible differences in faecal microbiota between men with a low serum enterolactone concentration and those with a high concentration, and to investigate the impact of a synbiotic mixture on serum enterolactone concentration in men with a low concentration. We compared faecal microbiota between ten men with the lowest serum enterolactone concentration and ten men with the highest concentration at recruitment ( $n$ 84). Furthermore, we carried out a randomised, double-blind, placebo-controlled, cross-over intervention study (6-week intervention periods and 4-week washout period) to investigate the impact of a synbiotic mixture (two Lactobacillus strains, one Bifidobacterium strain, one Propionibacterium strain and galacto-oligosaccharides $(32 \mathrm{~g} / \mathrm{l})$ ) on serum enterolactone concentration in fifty-two men who had a concentration $<20 \mathrm{nmol} / \mathrm{l}$. Serum sensitive C-reactive protein (CRP) concentration was measured at the end of the first intervention period. Men with a low serum enterolactone concentration when compared with those with a high concentration had less faecal bacteria, especially those belonging to the Lactobacillus-Enterococcus group (median 8.2 (interquartile range 7.8-8.4) $\log _{10}$ colony-forming units/g $v$. median 8.8 (interquartile range $8.5-8.9) \log _{10}$ colony-forming units $/ \mathrm{g}, P=0.009$ ). The synbiotic mixture that was used did not have a significant effect on serum enterolactone (synbiotic $v$. placebo ratio 0.96 (95\% CI $0.76,1 \cdot 22), P=0 \cdot 724)$ or serum sensitive CRP (synbiotic $v$. placebo ratio $0.99(95 \%$ CI $0.74,1.33), P=0.954)$ concentration. Men with a low serum enterolactone concentration harbour less colonic bacteria, especially those belonging to the Lactobacillus-Enterococcus group. A synbiotic mixture does not increase serum enterolactone concentration.

\section{Key words: Faecal microbiota: Serum enterolactone: Sensitive C-reactive protein: Synbiotic mixture}

Low serum enterolactone concentration is an independent risk factor of acute coronary events and death for CHD and CVD in men ${ }^{(1,2)}$. The inhibition of lipid peroxidation ${ }^{(3,4)}$, up-regulation of hepatic LDL receptor activity ${ }^{(5)}$, inhibition of lipoprotein uptake by macrophages ${ }^{(6)}$ and oestrogen-like effect $^{(7)}$ are the proposed mechanisms behind the protective effect of enterolactone. Enterolactone is produced by intestinal metabolism of food lignans such as matairesinol, secoisolariciresinol, pinoresinol and lariciresinol ${ }^{(8-10)}$, non-energetic, phenolic plant compounds abundantly found in whole-grain cereals, vegetables and some fruits and berries ${ }^{(10,11)}$. Enterolactone is the main circulating product of intestinal lignan metabolism ${ }^{(10)}$ and has been proposed to be more potent in terms of biological properties than the parent compounds ${ }^{(12)}$. Although it is possible that some lignan-metabolising enzymes are present in the intestinal brush border ${ }^{(13,14)}$, intestinal microbiota, especially those in the colon, are mainly responsible for the metabolism of lignans and are major determinants of serum enterolactone concentration ${ }^{(12,15)}$. Enterolactone formation requires a network of sequential reactions involving

Abbreviations: CRP, C-reactive protein; GOS, galacto-oligosaccharides; sCRP, sensitive C-reactive protein.

*Corresponding author: Dr R. Holma, fax +358 9191 25364, email reetta.holma@helsinki.fi 
several species, most of which are common members of dominant bacterial groups in the human intestine ${ }^{(12)}$. For enterolactone formation to take place, there need to be sufficient amounts of both lignan and bacteria capable of forming enterolactone.

The serum concentration of high-sensitivity C-reactive protein (CRP), a predictor of cardiovascular events among healthy individuals ${ }^{(16)}$, has been reported in healthy participants to be inversely correlated with the total faecal microbial counts $^{(17)}$ and bacterial populations with high DNA guanine + cytosine contents $^{(18)}$. Furthermore, reductions in CRP concentration have been associated with increases in the bacterial counts of lactobacilli and bifidobacteria, but not with those in the counts of any other bacteria, in the faeces of healthy humans ${ }^{(19)}$. Intestinal microbes can induce a cascade of immunological events either through pathogen-associated molecular patterns on their surface recognised by Toll-like receptors in the gut epithelial cells and antigen-presenting cells $^{(20)}$ or through products of their metabolism, such as SCFA $^{(21,22)}$.

In the present study, we compared faecal microbiota between men with the lowest serum enterolactone concentration and those with the highest concentration at recruitment and carried out a randomised, double-blind intervention study with a cross-over design to investigate whether a synbiotic mixture, consisting of a specific probiotic combination (two Lactobacillus strains, one Bifidobacterium strain and one Propionibacterium strain) and prebiotic galacto-oligosaccharides (GOS), could elevate serum enterolactone concentration in men who have a low serum enterolactone concentration. The effect of the synbiotic mixture on serum sensitive C-reactive protein (sCRP) concentration was also investigated. The consumption of lignan-rich foods was monitored during the intervention periods using a FFQ. This particular synbiotic combination was chosen on the basis of our previous openlabel study with sequential 2 -week interventions ${ }^{(23)}$, which suggested that in a subgroup of men with a serum enterolactone concentration $<20 \mathrm{nmol} / 1$ at run-in, the mean serum enterolactone concentration was doubled at the end of the intervention periods with this combination. In men with a serum enterolactone concentration $\geq 20 \mathrm{nmol} / \mathrm{l}$ at run-in, no change in the mean serum enterolactone concentration was observed. Therefore, we hypothesised that this synbiotic combination would increase serum enterolactone concentration in men with a value $<20 \mathrm{nmol} / 1$ when given for a longer period of time. To our knowledge, there are no other studies on the effects of probiotics, prebiotics or synbiotics on serum enterolactone concentration.

\section{Materials and methods}

\section{Participants}

Men, aged 20-65 years, were recruited from the personnel of four industrial companies in Southern Finland by an advertisement. The serum enterolactone concentration of eighty-four volunteers was determined at recruitment. In part I of the study, 'Cross-sectional comparison of faecal microbiota in groups with low and high serum enterolactone concentrations', ten men with the lowest serum enterolactone concentration and ten men with the highest concentration were selected (Table 1). In part II, 'Cross-over intervention study', those who had a serum enterolactone concentration $<20 \mathrm{nmol} / 1$, in total fifty-six of the eighty-four volunteers, were selected. Among them, four withdrew from the study before it begun, resulting in fifty-two participants in part II (Table 1). Before recruitment into the study, the participants of both parts were interviewed for illness, medication, diet, smoking and alcohol use. Exclusion criterion was antibiotic treatment for 2 months before the interventions. All the participants consumed an omnivorous diet, except for one, who consumed a lactovegetarian diet. The present study was conducted according

Table 1. Characteristics of the study participants at recruitment in part I, 'Cross-sectional comparison of faecal microbiota in groups with high and low enterolactone concentrations' and in part II, 'Cross-over intervention study'

(Mean values and ranges)

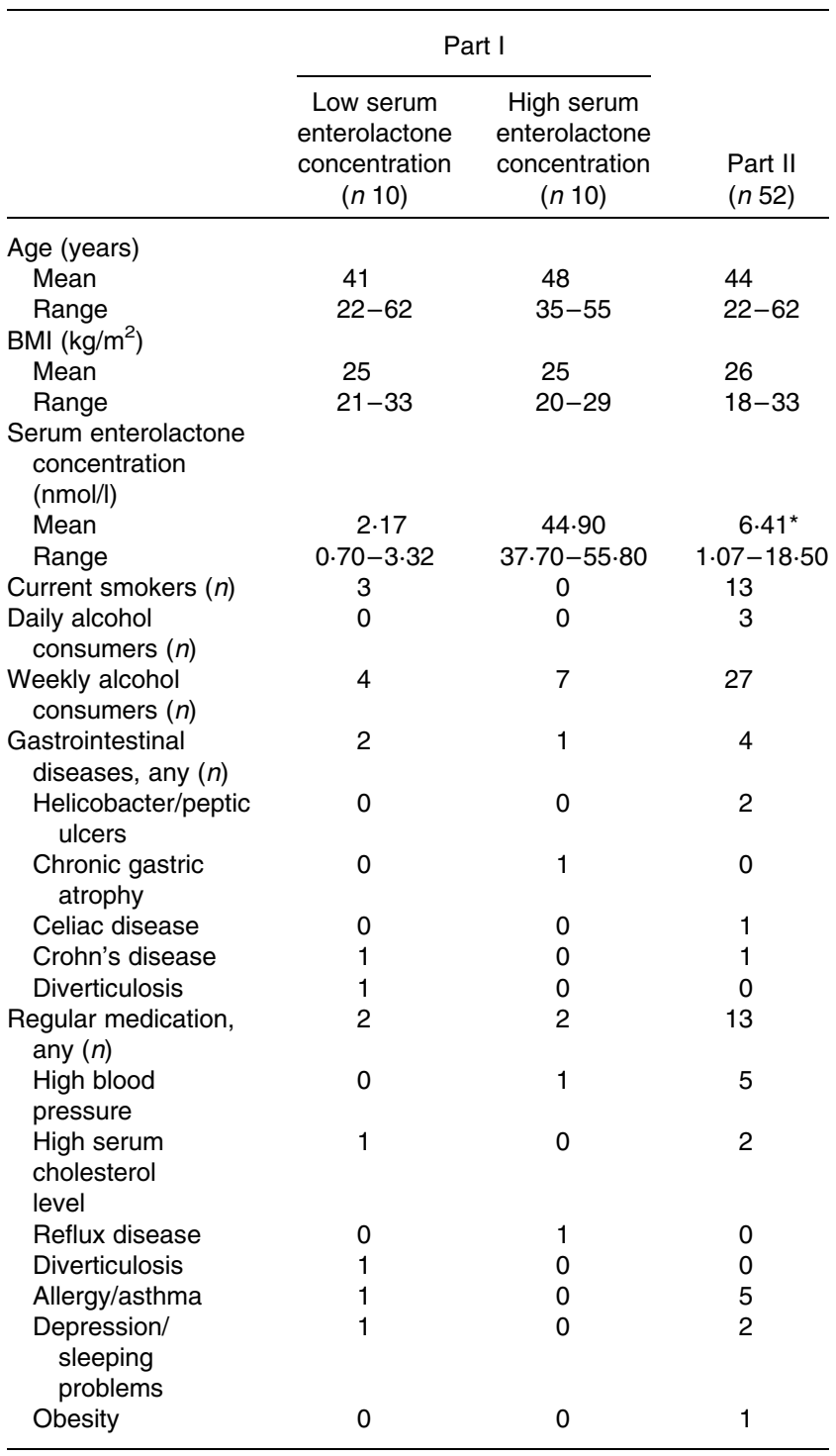

*Geometric mean. 
to the guidelines laid down in the Declaration of Helsinki, and all procedures involving human subjects were approved by the Ethics Committee of the Hospital District of Helsinki and Uusimaa. Written informed consent was obtained from all the subjects.

\section{Interventions}

The intervention study was a randomised, double-blind, placebo-controlled, two-period cross-over trial with 6-week treatment periods and a 4 -week washout period. Group A consumed one bacterial capsule together with $0 \cdot 1$ litres of GOS juice (a three-fruit juice (grape, orange and passion fruit), GOS $32 \mathrm{~g} / \mathrm{l}$ ) daily during the first treatment period, followed by consumption of placebo capsules and a placebo juice (fruit juice without GOS) during the second treatment period, and group $\mathrm{B}$ received the treatments in the reverse order. The participants were randomised into group A or group $\mathrm{B}$ according to a computer-generated random permuted-block method and a block size of four subjects. The participants were instructed to take the capsules and the juice once daily with the first meal of the day.

Bacterial capsules contained a probiotic mixture of two Lactobacillus rhamnosus strains, GG (ATCC 53103, 5.9 × $10^{10}$ colony-forming units (CFU)/1) and LC705 (DSM7061, $\left.1.3 \times 10^{11} \mathrm{CFU} / \mathrm{l}\right)$, one Bifidobacterium strain, Bifidobacterium breve Bb99 (DSM 13692, 5.7 × 109 CFU/1), and one Propionibacterium strain, Propionibacterium freudenreichii ssp. shermanii JS (DSM7067, $1 \times 10^{11} \mathrm{CFU} / \mathrm{l}$ ). In addition to the bacteria, the capsules contained microcrystalline cellulose and gelatine as a filler and were lactose free. The placebo capsules were of identical composition but without the bacteria.

Before the start of the intervention study, the participants were instructed not to consume products containing any lactic acid bacteria considered probiotic, including certain type of cheeses containing the investigated probiotics L. rhamnosus $\mathrm{LC} 705$ and $P$. freudenreichii ssp. shermanii JS (Emmental and Polar), seeds and nuts during the whole study period starting 4 weeks before the start of the study. The participants were instructed not to change their ordinary diet and habits in any other way.

\section{Questionnaires}

At recruitment, a FFQ was used to gain information about the participants' dietary habits during the previous 2 months. There were six consumption frequency options: not once; one to three times a month; one to two times a week; three to five times a week; every day or almost every day; several times a day. The participants were asked to fill in the portion size in numbers. The questionnaire included questions on a total of fifty-six foods and food groups. In part II, more detailed FFQ were administered after both treatment periods in order to monitor the consumption of lignan-rich foods, such as cereals, fruits, berries, vegetables, nuts and seeds, and of products containing probiotic bacteria during the study. The questions concerned foods consumed during the previous 4 weeks, and there were three consumption frequency options: $X$ times a day/week/month. The participants were asked to fill in the frequency in numbers in only one frequency option. They were asked to fill in the portion size in numbers. The questionnaire included a total of eighty questions. In part II, the participants were asked about the use of antibiotics after each treatment period. After the second treatment period, the participants filled in a questionnaire about their gastrointestinal symptoms (stomach ache, abdominal distension, flatulence, heartburn, loose stools and hard stools: $0=$ no symptoms; $1=$ a few; $2=$ moderately; $3=$ a lot) during that period and also in their everyday life.

\section{Blood and faecal samples}

Blood samples for enterolactone analyses were collected at recruitment and four times during the experiment in part II, at the beginning and end of both the treatment periods. Serum was separated from the blood samples not more than $2 \mathrm{~h}$ after sampling. The samples were centrifuged and stored at $-20^{\circ} \mathrm{C}$ until analysis. Enterolactone analyses were carried out using a time-resolved fluoroimmunoassay ${ }^{(24,25)}$. Serum sCRP concentration was determined in part II at the beginning and end of the first treatment period using a highly sensitive immunoturbidimetric assay (Tina-quant CRP high-sensitive assay reagent, Roche Hitachi 912 analyser; Roche Diagnostics $\mathrm{GmbH})$.

Faecal samples for the microbiological analysis were collected at recruitment to study the differences in faecal microbiota in part I between men with the lowest serum enterolactone concentration ( $n$ 10) and those with the highest concentration ( $n$ 10). Men were instructed to freeze the faecal samples immediately and to keep them frozen until taken in a cooler with ice packs to the study centre, where they were immediately stored at $-70^{\circ} \mathrm{C}$ until analysis.

Fluorescence in situ hybridisation analysis of faecal samples was carried out as described previously ${ }^{(26)}$. Bacterial cells were counted visually with an epifluorescence microscope, examining a minimum of fifteen fields in each sample. DAPI (4',6-diamidino-2-phenylindole) dye was used for counting total bacteria. Oligonucleotide probes used were Cy3-labelled Bfra602 (Bacteroides fragilis group), Bdis656 (Bacteroides distasonis), Erec482 (Eubacterium rectale-Clostridium coccoides group), Bif164 (bifidobacteria), Chis150 (Clostridium histolyticum) and Lab158 (lactobacilli and enterococci).

For bacterial counts analysed by cultivation, faecal samples were homogenised in 1:10 with the Wilkins-Chalgren broth (Oxoid Limited) in an anaerobic chamber. Subsequently, tenfold serial dilutions were plated on appropriate agars. Total anaerobes were cultivated anaerobically on BrainHeart Infusion Agar (Oxoid) at $37^{\circ} \mathrm{C}$ for $5 \mathrm{~d}$ and total aerobes on the same agar aerobically for $3 \mathrm{~d}$. Total lactobacilli were cultivated on De Man Rogosa Sharpe (MRS) agar for $3 \mathrm{~d}$, bifidobacteria on Raffinose agar for $2 \mathrm{~d}$ and L. rhamnosus GG as well as Lactobacillus 705 on MRS Agar supplemented with vancomycin for $3 \mathrm{~d}$, all anaerobically at $37^{\circ} \mathrm{C}$, as described previously ${ }^{(23)}$. B. fragilis was cultivated anaerobically on Bacteroides Bile Esculin Agar at $37^{\circ} \mathrm{C}$ for $2-4 \mathrm{~d}$. 
Sulphite-reducing clostridia were cultivated anaerobically on Ferrous Sulphite Agar at $37^{\circ} \mathrm{C}$ for $1-2 \mathrm{~d}$.

\section{Statistical analysis}

Part I. 'Cross-sectional comparison of faecal microbiota in groups with low and high serum enterolactone concentrations'. Faecal bacterial plate and fluorescence in situ hybridisation counts were the primary variables. They were logarithmically $\left(\log _{10}\right)$ transformed and are expressed as $\log _{10} \mathrm{CFU} / \mathrm{g}$. The Mann-Whitney $U$ test was used as a primary analysis to compare the groups of men with low and high serum enterolactone concentrations. ANCOVA was used as a secondary analysis in order to control the possible effect of the intake of lignan-rich foods. Based on the consumption of twenty lignan-rich foods given by FFQ, two summary statistics were derived. First, the total number of lignan-rich foods consumed was calculated. In addition, the total consumption was roughly estimated (consumption frequency $\times$ portion size). These two variables were included as covariates in separate ANCOVA models, in which the bacterial counts as $\log _{10} \mathrm{CFU} / \mathrm{g}$ were compared between groups with a high serum enterolactone concentration and those with a low concentration.

Part II. 'Cross-over intervention study'. All data were analysed based on the intention-to-treat population, which included all fifty-two randomised men who were enrolled in the study and had at least one follow-up measurement of serum enterolactone or sCRP concentration. In this population, three men were on an antibiotic course during the second treatment period and discontinued the study. Their serum enterolactone and SCRP values at the end of the second treatment period were set as missing values. Serum enterolactone and SCRP concentrations were the primary variables. As the distributions were skewed to the right, they were logarithmically $\left(\log _{e}\right)$ transformed before analysis. Serum enterolactone concentration was measured at the beginning and end of treatment periods 1 and 2. The repeated-measures ANOVA for cross-over designs was used to analyse the concentrations at the end of the treatment periods and to analyse the within-period changes. The results are given as ratios (synbiotic:placebo) and mean difference (synbiotic - placebo) with $95 \%$ CI, respectively. The within-period changes (enterolactone concentration at the end of the treatment period minus that at the beginning of the treatment period) were calculated using untransformed values. The period and carryover effects were non-significant for both analyses.

Serum sCRP concentration was measured at the end and beginning of treatment period 1 . The synbiotic and placebo groups were compared at the end of the treatment period using ANCOVA in which the value at the beginning was included as a covariate. The result is given as a ratio (synbiotic:placebo) with 95\% CI. In addition, the absolute within-period changes were analysed using ANOVA.

The percentage of users of different lignan-rich foods and the number of weekly servings were compared between treatment periods 1 and 2 in order to assess the stability of diet during the study period. Changes in the percentage of users and the number of weekly servings were analysed using the McNemar test and Wilcoxon signed-rank test, respectively.

Gastrointestinal symptoms (scores 0-3) during everyday life and during treatment period 2 were assessed. Logistic regression analysis was used to compare the treatment groups with respect to any grade (1-3) of symptoms during treatment. The ordinary symptoms of any grade were included as a categorical covariate. In addition, score $(0-21)$ of the sum of symptoms $=$ stomach ache + abdominal distension + flatulence + heartburn + diarrhoea + loose stools + hard stools) was calculated. The treatment groups were compared using ANOVA and ANCOVA, in which the sum of symptom scores during everyday life was included as a continuous covariate.

All tests were carried out as two sided, and $P$ values $<0.05$ were considered statistically significant. Statistical analysis was carried out using IBM SPSS version 20.0 (SPSS, Inc.).

\section{Results}

Part I

Faecal bacterial counts in participants with low and high serum enterolactone concentrations. The mean (range) serum enterolactone concentrations of the ten lowest and ten highest concentrations at recruitment were $2 \cdot 17(0 \cdot 70-$ 3.32 ) and $44.90(37 \cdot 70-55 \cdot 80) \mathrm{nmol} / \mathrm{l}$, respectively (Table 1$).$ Faecal bacterial counts of these two groups are given in Table 2. The participants with a low serum enterolactone concentration had significantly less total bacteria, both aerobic and anaerobic, in faeces than those with a high serum enterolactone concentration. The difference in medians $6.2 v .7 \cdot 7$ in the counts of aerobic bacteria in the groups with low and high serum enterolactone concentrations indicated approximately thirtyfold difference in the medians of untransformed bacterial counts. Similarly, the difference in medians $8.4 \mathrm{v}$. $9 \cdot 2$ in the counts of anaerobic bacteria indicated more than sixfold difference in untransformed bacterial counts. The Lactobacillus-Enterococcus group was the only one that was found to have significantly different counts between the two participant groups, with approximately threefold larger medians of untransformed bacterial counts in those with a high serum enterolactone concentration when compared with those with a low concentration. In the low-enterolactone concentration group, one participant had Crohn's disease and one had diverticulosis, and in the high-enterolactone concentration group, one participant had chronic gastric atrophy (Table 1). Their exclusion from the analysis did not affect the results obtained, so the results are presented for all the participants. None of these twenty men reported constipation.

Even when the results were adjusted in separate models for the total number of lignan-rich foods consumed and the total consumption of lignan-rich foods during the previous 2 months, the total bacterial counts and the anaerobic bacterial counts were significantly higher in the group with a high serum enterolactone concentration $(P=0.001$ and 0.002 for total bacteria; $P=0.012$ and 0.016 for anaerobic bacteria, respectively). The counts of the Lactobacillus-Enterococcus group were also significantly higher in the group with a 
Table 2. Faecal bacterial plate and fluorescence in situ hybridisation (FISH) counts in groups with low and high serum enterolactone concentrations at recruitment in part I, 'Cross-sectional comparison of faecal microbiota in groups with high and low enterolactone concentrations'

(Medians and interquartile ranges (IQR))

\begin{tabular}{|c|c|c|c|c|c|}
\hline & \multicolumn{2}{|c|}{$\begin{array}{c}\text { Low serum } \\
\text { enterolactone } \\
\text { concentration }(n 10)\end{array}$} & \multicolumn{2}{|c|}{$\begin{array}{c}\text { High serum } \\
\text { enterolactone } \\
\text { concentration }(n 10)\end{array}$} & \multirow[b]{2}{*}{$P^{*}$} \\
\hline & Median & IQR & Median & IQR & \\
\hline \multicolumn{6}{|l|}{$\mathrm{FISH}\left(\log _{10} \mathrm{CFU} / \mathrm{g}\right)$} \\
\hline Total bacteria & 11.5 & $11 \cdot 3-11 \cdot 6$ & $11 \cdot 8$ & $11 \cdot 7-12 \cdot 0$ & 0.001 \\
\hline Bacteroides fragilis-Bacteroides distasonis & $10 \cdot 7$ & $10 \cdot 6-11 \cdot 0$ & 11.0 & $10 \cdot 8-11 \cdot 1$ & 0.14 \\
\hline Eubacterium rectale-Clostridium coccoides & $10 \cdot 6$ & $10 \cdot 6-11 \cdot 0$ & $11 \cdot 1$ & $10 \cdot 8-11 \cdot 3$ & 0.05 \\
\hline Bifidobacteria & $10 \cdot 0$ & $9 \cdot 3-10 \cdot 5$ & $10 \cdot 2$ & $9 \cdot 7-10 \cdot 3$ & 0.63 \\
\hline Clostridium histolyticum & 8.5 & $8.3-8.6$ & 8.4 & $8.0-8.9$ & 0.91 \\
\hline Lactobacilli/enterococci & $8 \cdot 2$ & $7 \cdot 8-8 \cdot 4$ & $8 \cdot 8$ & $8.5-8.9$ & 0.009 \\
\hline \multicolumn{6}{|l|}{ Plating $\left(\log _{10} \mathrm{CFU} / \mathrm{g}\right)$} \\
\hline Bifidobacteria & $8 \cdot 6$ & $7 \cdot 7-9 \cdot 0$ & $9 \cdot 0$ & $8 \cdot 3-9 \cdot 3$ & 0.35 \\
\hline Anaerobic bacteria & 8.4 & $7 \cdot 0-8 \cdot 7$ & $9 \cdot 2$ & $9.1-9.5$ & 0.002 \\
\hline Aerobic bacteria & $6 \cdot 2$ & $6 \cdot 0-7.4$ & $7 \cdot 7$ & $7 \cdot 0-8 \cdot 7$ & 0.029 \\
\hline Bacteroides fragilis & $6 \cdot 6$ & $5 \cdot 0-6 \cdot 9$ & $6 \cdot 6$ & $6 \cdot 1-7 \cdot 2$ & 0.32 \\
\hline Lactobacilli, total & 5.4 & $5 \cdot 1-6 \cdot 2$ & 5.9 & $5 \cdot 0-7 \cdot 0$ & 0.58 \\
\hline Lactobacillus LC705 & $5 \cdot 2$ & $3 \cdot 0-5 \cdot 5$ & $5 \cdot 0$ & $4 \cdot 3-5 \cdot 5$ & 0.97 \\
\hline Lactobacillus GG & 4.4 & $3 \cdot 6-5 \cdot 8$ & 4.0 & $3 \cdot 6-6 \cdot 2$ & 0.80 \\
\hline Sulphite-reducing clostridia & $4 \cdot 1$ & $3 \cdot 8-5 \cdot 0$ & 4.6 & $4 \cdot 0-5 \cdot 5$ & 0.28 \\
\hline
\end{tabular}

high serum enterolactone concentration when the results were adjusted for the number of lignan-rich foods consumed $(P=0 \cdot 029)$. However, when the consumption of lignan-rich foods was included as a covariate, the difference between these groups was non-significant $(P=0 \cdot 124)$, although the adjusted means of $8.7 v .8 .3$ in the log scale indicated a $2 \cdot 5$-fold difference in the original counts. The aerobic bacterial counts were only marginally significantly higher in the group with a high serum enterolactone concentration when the results were adjusted for the number of lignan-rich foods consumed and for the consumption of lignan-rich foods $(P=0.056$ and 0.086). After adjustment for the number of lignan-rich foods consumed, the adjusted means $7.6 v .6 .5$ in the log scale indicated more than tenfold difference in the original counts. Similarly, after adjustment for the total consumption of lignan-rich foods, the adjusted means $7.7 v .6 .5$ indicated more than tenfold difference.

\section{Part II}

Compliance. During the second treatment period, three men were withdrawn from the study because of the use of antibiotics (two during placebo treatment and one during synbiotic treatment). Thus, forty-nine men completed the study. None of them used antibiotics during the study period. After treatment period 2, one of them failed to reply to the FFQ and gastrointestinal symptom questionnaire. Compliance with the interventions was good, with only five men being reported as acting contrary to the instructions given at some point during the study period. Mostly, these participants did not comply with the instructions for $1-2 \mathrm{~d}$ during the 6 -week periods, but during the intervention periods, one participant did not consume the juice with GOS for ten consecutive days and one participant for three consecutive days. In addition, one participant did not consume the juice with GOS or the capsules for five consecutive days during the intervention periods. Instructions to avoid the consumption of certain products were more often disobeyed, but concerned mostly one to two incidents during the 6-week intervention periods. The consumption of products contrary to instructions was approximately the same during the two interventions. However, during placebo treatment, one participant consumed L. rhamnosus GG-containing sour milk every day and one participant Lactobacillus reuteri-containing yogurt once weekly. The exclusion of these two men from the analysis of main variables did not change the results, so the results are presented for all the participants. No other products containing probiotics were reported. During placebo treatment, one person reported to have lost $7 \mathrm{~kg}$ weight.

Dietary habits. There were no significant differences in the percentage of users of different lignan-rich foods between treatment periods 1 and 2 (Table 3). The users of lignan-rich foods or food groups remained the same in at least $70 \%$ of the study population between treatment periods 1 and 2. No significant differences were detected in the weekly servings of different lignan-rich foods either, except for beer, the consumption of which was slightly higher during treatment period 1 than during treatment period 2 (median 0.75 v. 0.53 litres weekly, respectively, $P=0 \cdot 004)$.

Foods whose consumers were at least $90 \%$ the same individuals when comparing the two treatment periods are not reported in Table 3. These were rye bread, fruits, vegetables, potatoes, onions and beer. Foods that were used by an insufficient number of users to conduct statistical tests of the number of weekly servings are not reported in Table 3 either. 
Table 3. Users and number of servings of different lignan-rich foods during treatment periods 1 and 2 according to a FFQ in part II, 'Cross-over intervention study'

(Medians and interquartile ranges (IQR))

\begin{tabular}{|c|c|c|c|c|c|c|c|c|}
\hline & & & & \multicolumn{4}{|c|}{ Number of servings per week } & \multirow[b]{3}{*}{$P \dagger$} \\
\hline & \multicolumn{3}{|c|}{ Users ( $n$ 48) } & \multicolumn{2}{|c|}{ Period 1} & \multicolumn{2}{|c|}{ Period 2} & \\
\hline & Period $1(\%)$ & Period $2(\%)$ & $P^{*}$ & Median & IQR & Median & IQR & \\
\hline White wheat bread $(50 \mathrm{~g}) \ddagger$ & 75 & 75 & 1.00 & 1.5 & $0.1-5 \cdot 8$ & 1.5 & $0.1-5.9$ & 0.37 \\
\hline Graham roll $(30 \mathrm{~g}) \ddagger$ & 88 & 94 & 0.45 & $7 \cdot 5$ & $2 \cdot 7-15 \cdot 6$ & $6 \cdot 3$ & $2 \cdot 1-15 \cdot 8$ & 0.55 \\
\hline Porridge (plateful) & 50 & 52 & 1.00 & 0.1 & $0.0-1.4$ & 0.3 & $0 \cdot 0-2 \cdot 0$ & 0.19 \\
\hline Berries $(\mathrm{dl}) \ddagger$ & 73 & 73 & 1.00 & 0.9 & $0.0-2 \cdot 3$ & 0.6 & $0 \cdot 0-2 \cdot 0$ & 0.77 \\
\hline Juice (dl) & 75 & 69 & 0.58 & 7.5 & $0.1-15 \cdot 0$ & 4.0 & $0.0-14.1$ & 0.23 \\
\hline Juice drink (dl)‡ & 54 & 54 & 1.00 & 0.8 & $0.0-3.9$ & 1.0 & $0 \cdot 0-7 \cdot 2$ & 1.00 \\
\hline Fool (dl)‡ & 54 & 50 & $0 \cdot 77$ & 0.3 & $0.0-2.9$ & 0.1 & $0 \cdot 0-2 \cdot 8$ & 0.33 \\
\hline Peas, lentils and beans $(\mathrm{dl}) \ddagger$ & 65 & 63 & 1.00 & 0.3 & $0.0-0.9$ & 0.3 & $0.0-1.0$ & 0.55 \\
\hline Pea soup (plateful) & 71 & 71 & 1.00 & 0.3 & $0.0-0.5$ & 0.3 & $0.0-0.5$ & 0.44 \\
\hline Garlic (clove) & 67 & 67 & 1.00 & 0.5 & $0.0-2 \cdot 0$ & 1.0 & $0 \cdot 0-2 \cdot 0$ & 0.67 \\
\hline Black tea (dl) & 69 & 77 & 0.34 & 3.5 & $0.0-10 \cdot 9$ & 3.5 & $0.3-14.8$ & 0.46 \\
\hline Red wine (glass) & 77 & 69 & 0.34 & 0.9 & $0.3-2 \cdot 0$ & 1.0 & $0.0-2 \cdot 0$ & 0.94 \\
\hline
\end{tabular}

\section{* McNemar's test.}

† Wilcoxon signed-rank test.

$\ddagger$ Combined variable: white wheat bread= white wheat bread or roll; graham roll = graham bread or roll, yeast bread or mixed-grain bread; juice drink $=$ homemade or commercial juice drink; fool = berry soup or fool, or fruit fool.

These were rye bran, any bran (rye, oat or wheat), muesli, breakfast cereals, berry porridge, berry quark, berry jam, dried fruits, pickles, nuts, almonds, seeds (linseeds, sesame seeds or sunflower seeds), germs, soya flour, green tea, black tea, whiskey and white wine. Their consumers were at least $70 \%$ the same individuals when comparing the two treatment periods, and no significant differences were found.

Gastrointestinal symptoms. The occurrence of gastrointestinal symptoms (stomach ache, abdominal distension, flatulence, heartburn, loose stools and hard stools) during treatment period 2 ( $n 25$ given synbiotics; $n 23$ given placebo) was not significantly different between the two treatment groups (data not shown). The differences between the treatment groups were not significant when the symptoms in their everyday life were or were not included as a covariate.

Serum enterolactone and sensitive C-reactive protein concentrations. Synbiotic treatment did not have a significant effect on serum enterolactone concentration (Table 4;
Fig. 1). There was no significant difference in serum sCRP concentration between synbiotic and placebo treatment groups (Table 4).

\section{Discussion}

The aims of the present study were to compare faecal microbiota between men with a low serum enterolactone concentration and those with a high concentration and to investigate whether a specific synbiotic mixture affects serum enterolactone and sCRP concentrations in men who have a low serum enterolactone concentration. The total counts of bacteria and those belonging to the LactobacillusEnterococcus group in faeces were significantly lower in participants with a low serum enterolactone concentration than in those with a high concentration. However, the particular synbiotic mixture used in the present study had no significant effect on serum enterolactone and sCRP

Table 4. Serum enterolactone and sensitive C-reactive protein (SCRP) concentrations ${ }^{\star}$ in part II, 'Cross-over intervention study'

(Geometric means for concentrations and means for changes and $95 \%$ confidence intervals)

\begin{tabular}{|c|c|c|c|c|c|c|c|}
\hline & \multicolumn{2}{|c|}{ Synbiotics } & \multicolumn{2}{|c|}{ Placebo } & \multicolumn{2}{|c|}{ Synbiotics $v$. placebo } & \multirow[b]{2}{*}{$P$} \\
\hline & Mean & $95 \% \mathrm{Cl}$ & Mean & $95 \% \mathrm{Cl}$ & Mean & $95 \% \mathrm{Cl}$ & \\
\hline \multicolumn{8}{|l|}{ Enterolactone $(\mathrm{nmol} / \mathrm{l})$} \\
\hline At the end of the intervention period $\dagger$ & 6.93 & $5.44,8.83$ & $7 \cdot 23$ & $5.50,9.51$ & $0.96 \ddagger$ & $0.76,1.22$ & $0.72 \S$ \\
\hline Change from the beginning\| & -3.76 & $-7.06,-0.47$ & -1.88 & $-3.82,0.06$ & -1.98 & $-5.41,1.64$ & $0.29 \S$ \\
\hline \multicolumn{8}{|l|}{$\mathrm{sCRP}(\mathrm{mg} / \mathrm{l})$} \\
\hline At the end of treatment period 19 & 0.88 & $0.72,1.08$ & 0.88 & $0.72,1.09$ & $0.99 \ddagger$ & $0.74,1.33$ & $0.95^{\star *}$ \\
\hline Change from the beginning & 0.06 & $-0.53,0.65$ & 0.04 & $-0.42,0.49$ & 0.02 & $-0.72,0.75$ & $0.95 \dagger \dagger$ \\
\hline
\end{tabular}

*Enterolactone and sCRP concentrations were logarithmically transformed before analysis.

†Only those whose enterolactone concentrations were analysed at the end of both the treatment periods were included ( $n$ 47).

$\ddagger$ Values are ratios.

$\S$ ANOVA for repeated measures.

II Only those whose enterolactone concentrations were analysed at the beginning and end of both the treatment periods were included ( $n$ 40).

I Only those whose sCRP concentrations were analysed both at the beginning and end of period 1 were included (synbiotics $n$ 23; placebo $n$ 22).

${ }^{* *}$ ANCOVA, where the value at the beginning was included as a covariate. Results are given as adjusted geometric means and $95 \% \mathrm{Cl}$.

††ANOVA. 




Fig. 1. Serum enterolactone concentration in part II, 'Cross-over intervention study'. Group A (O) received synbiotics during treatment period 1 and placebo during treatment period $2(n 24)$ and group B $(\bullet)$ received placebo during treatment period 1 and synbiotics during treatment period 2 ( $n$ 25). The dotted line indicates the washout period. Values are geometric means, with $95 \% \mathrm{Cl}$ represented by vertical bars.

concentrations. The consumption of lignan-rich foods appeared to remain stable during the intervention periods.

To our knowledge, this is the first study to compare faecal microbiota between men with a high serum enterolactone concentration and those with a low concentration. The faecal counts of anaerobic and aerobic bacteria were approximately six and thirty times higher, respectively, in those with a high serum enterolactone concentration than in those with a low concentration. This is not surprising since enterolactone is produced by intestinal microbiota ${ }^{(8-10)}$ and oral antimicrobial use decreases serum enterolactone concentration according to an epidemiological study ${ }^{(15)}$. The Lactobacillus-Enterococcus group was the only one to exhibit a significant difference between the two participant groups in the present study. It was found approximately three times more in men with a high serum enterolactone concentration than in those with a low concentration. Enterococcus faecalis strain PDG-1, a strain isolated from human faeces, has been reported to be highly capable of transforming pinoresinol to lariciresinol, the first step in the synthesis of enterolactone from pinoresinol ${ }^{(27)}$. Lactobacilli, on the other hand, have not been reported to be involved in enterolactone formation. However, human faecal lactobacilli, as well as enterococci, have $\beta$-glucosidase activity $^{(28)}$ and, therefore, have the potential to hydrolyse plant lignans that are glucosides ${ }^{(14,27)}$. Since plating results did not show differences in the counts of lactobacilli, enterococci might be responsible for the difference in fluorescence in situ hybridisation counts of the Lactobacillus-Enterococcus group between men with a high serum enterolactone concentration and those with a low concentration.
It is possible that high counts of the Lactobacillus-Enterococcus group are not involved in enterolactone production, but merely reflect a characteristic that is also associated with a high serum enterolactone concentration such as a certain type of diet or bowel habits. Whole-grain wheat, for instance, when compared with wheat bran, has been reported to increase faecal counts of lactobacilli, as well as bifidobacteria ${ }^{(29)}$, and is also a dietary source of enterolactone precursors ${ }^{(11)}$. Constipation is associated with both increased serum enterolactone levels ${ }^{(30)}$ and alterations in the colonic microbiota ${ }^{(31)}$. None of the men in the present study reported constipation, ruling out the possibility of it being a major explanatory factor for the differences in faecal microbiota between the two groups.

The lack of an effect of the synbiotics on serum enterolactone concentration in the present intervention study is in agreement with a similar result obtained in an earlier synbiotic intervention study in postmenopausal women with or without a history of breast cancer ${ }^{(32)}$. Our recent results obtained in an open-label study also showed that a synbiotic combination similar to the one used in the present study does not significantly affect serum enterolactone concentration during a short intervention period in men with a normal serum enterolactone concentration ${ }^{(23)}$. In the previous study, the faecal counts of total lactic acid bacteria were not significantly increased during the consumption of this synbiotic mixture, although the counts of both the administered strains of lactobacilli were (as well as of total bifidobacteria and propionibacteria). It can, therefore, be speculated that the synbiotic mixture used in the present study did not increase the faecal lactobacilli counts in total, although modification at the strain level most probably occurred. Faecal bacteria were not assessed at the end of the intervention periods, which can be considered as a limitation of the present study. The administered probiotics, however, most probably survived through the gastrointestinal tract, since each of these probiotic bacteria has previously been detected in the faeces after administration of capsules similar to those used in the present study (without GOS) with metabolic effects in the colon ${ }^{(33)}$.

Previous studies have shown that most of the increase in serum enterolactone concentration occurs during the first 2-6 weeks of the intervention period with a lignan-rich diet, although it continues to increase until 12 weeks ${ }^{(34,35)}$. This suggests that the intervention of 6 weeks was probably long enough to detect the effect of the synbiotic intervention.

The results of the present study that the synbiotic mixture used had no significant effect on serum sCRP concentration are similar to those of a recent intervention using another synbiotic combination (three Lactobacillus strains, one Bifidobacterium strain, Streptococcus thermophilus and fructo-oligosaccharides) in healthy adults ${ }^{(36)}$. A synbiotic mixture the same as that used in the present study has increased serum CRP concentration in allergy-prone infants ${ }^{(37)}$, but a similar probiotic combination, with the only difference being in the Bifidobacterium strain used, without GOS did not affect serum CRP concentration in irritable bowel syndrome $^{(38)}$. Serum sCRP in adults without immunological disturbances is possibly resistant to modifications of intestinal microbiota by probiotics. 
In conclusion, men with a low serum enterolactone concentration have less faecal bacteria, especially those belonging to the Lactobacillus-Enterococcus group, than those with a high concentration. However, the synbiotic mixture, composed of two strains of lactobacilli and one strain of bifidobacteria and propionibacteria together with GOS, did not significantly affect serum enterolactone concentration in men with a low serum enterolactone concentration. This suggests that these strains participate in enterolactone synthesis, but do not colonise in sufficient amounts to have an impact, do not provoke a major modification in the resident microbiota participating in enterolactone synthesis, or simply are not taking part in enterolactone formation.

\section{Acknowledgements}

The present study was supported by Valio Limited, Helsinki, Finland, and the Finnish Funding Agency of Technology and Innovation. The authors' contributions are as follows: R. H. and R. A. K. analysed the data and wrote the paper; R. H. had primary responsibility for the final content; R. K., K. H., H. A., R. A. K. and H. V. designed and conducted the research; T. P. conducted the statistical analysis; R. K. directed the research. All authors read and approved the final manuscript. R. K. is Professor of Medical Nutrition Physiology at the Institute of Biomedicine, University of Helsinki, and a former employee of Valio Research Centre, Helsinki, Finland. R. A. K. and K. H. are employees of Valio Research Centre. R. H. and T. P. received compensation from Valio Research Centre. H. V. and H. A. have no conflicts of interest.

\section{References}

1. Vanharanta M, Voutilainen S, Lakka TA, et al. (1999) Risk of acute coronary events according to serum concentrations of enterolactone: a prospective population-based case-control study. Lancet 354, 2112-2115.

2. Vanharanta M, Voutilainen S, Rissanen TH, et al. (2003) Risk of cardiovascular disease-related and all-cause death according to serum concentrations of enterolactone - Kuopio Ischaemic Heart Disease Risk Factor Study. Arch Intern Med 163, 1099-1104.

3. Kitts DD, Yuan YV, Wijewickreme AN, et al. (1999) Antioxidant activity of the flaxseed lignan secoisolariciresinol diglycoside and its mammalian lignan metabolites enterodiol and enterolactone. Mol Cell Biochem 202, 91-100.

4. Vanharanta M, Voutilainen S, Nurmi T, et al. (2002) Association between low serum enterolactone and increased plasma $\mathrm{F}_{2}$-isoprostanes, a measure of lipid peroxidation. Atherosclerosis 160, 465-469.

5. Owen AJ, Roach PD \& Abbey M (2004) Regulation of low-density lipoprotein receptor activity by estrogens and phytoestrogens in a HepG2 cell model. Ann Nutr Metab 48, 269-275.

6. Owen AJ \& Abbey M (2004) The effect of estrogens and phytoestrogenic lignans on macrophage uptake of atherogenic lipoproteins. Biofactors 20, 119-127.

7. Penttinen P, Jaehrling J, Damdimopoulos AE, et al. (2007) Diet-derived polyphenol metabolite enterolactone is a tissue-specific estrogen receptor activator. Endocrinology 148, 4875-4886.
8. Borriello SP, Setchell KDR, Axelson M, et al. (1985) Production and metabolism of lignans by the human faecal flora. J Appl Bacteriol 58, 37-43.

9. Heinonen S, Nurmi T, Liukkonen $\mathrm{K}$, et al. (2001) In vitro metabolism of plant lignans: new precursors of mammalian lignans enterolactone and enterodiol. J Agric Food Chem 49, 3178-3186.

10. Peterson J, Dwyer J, Adlercreutz H, et al. (2010) Dietary lignans: physiology and potential for cardiovascular disease risk reduction. Nutr Rev $\mathbf{6 8}, 571-603$.

11. Adlercreutz H (2007) Lignans and human health. Crit Rev Clin Lab Sci 44, 483-525.

12. Clavel T, Doré J \& Blaut M (2006) Bioavailability of lignans in human subjects. Nutr Res Rev 19, 187-196.

13. Rowland I, Faughnan M, Hoey L, et al. (2003) Bioavailability of phyto-oestrogens. Br J Nutr 89, Suppl. 1, S45-S58.

14. Clavel T, Henderson G, Engst W, et al. (2006) Phylogeny of human intestinal bacteria that activate the dietary lignan secoisolariciresinol diglucoside. FEMS Microbiol Ecol 55, 471-478.

15. Kilkkinen A, Pietinen P, Klaukka T, et al. (2002) Use of oral antimicrobials decreases serum enterolactone concentration. Am J Epidemiol 155, 472-477.

16. Ridker PM (2007) C-reactive protein and the prediction of cardiovascular events among those at intermediate risk. Moving an inflammatory hypothesis toward consensus. J Am Coll Cardiol 49, 2129-2138.

17. Tiihonen K, Ouwehand AC \& Rautonen N (2010) Effect of overweight on gastrointestinal microbiology and immunology: correlation with blood biomarkers. Br J Nutr 103, 1070-1078.

18. Brignardello J, Morales P, Diaz E, et al. (2010) Pilot study: alterations of intestinal microbiota in obese humans are not associated with colonic inflammation or disturbances of barrier function. Aliment Pharmacol Ther 32, 1307-1314.

19. Tzounis X, Rodriguez-Mateos A, Vulevic J, et al. (2011) Prebiotic evaluation of cocoa-derived flavanols in healthy humans by using a randomized, controlled, double-blind, crossover intervention study. Am J Clin Nutr 93, 62-72.

20. Tlaskalová-Hogenová H, Stepánková R, Hudcovic T, et al. (2004) Commensal bacteria (normal microflora), mucosal immunity and chronic inflammatory and autoimmune diseases. Immunol Lett 93, 97-108.

21. Sanderson IR (2007) Dietary modulation of GALT. $J$ Nutr 137, Suppl. 11, 2557S-2562S.

22. Seifert S \& Watzl B (2007) Inulin and oligofructose: review of experimental data on immune modulation. J Nutr 137, Suppl. 11, 2563S-2567S.

23. Kekkonen RA, Holma R, Hatakka K, et al. (2011) A probiotic mixture including galactooligosaccharides decreases fecal $\beta$-glucosidase activity but does not affect serum enterolactone concentration in men during a two-week intervention. J Nutr 141, 870-876.

24. Adlercreutz H, Wang GJ, Lapcik O, et al. (1998) Time resolved fluoroimmunoassay for plasma enterolactone. Anal Biochem 265, 208-215.

25. Stumpf K, Uehara M, Nurmi T, et al. (2000) Changes in the time-resolved fluoroimmunoassay of plasma enterolactone. Anal Biochem 284, 153-157.

26. Myllyluoma E, Ahlroos T, Veijola L, et al. (2007) Effects of anti-Helicobacter pylori treatment and probiotic supplementation on intestinal microbiota. Int J Antimicrob Agents 29, $66-72$.

27. Xie LH, Akao T, Hamasaki K, et al. (2003) Biotransformation of pinoresinol diglucoside to mammalian lignans by human intestinal microflora, and isolation of Enterococcus faecalis strain PDG-1 responsible for the transformation of 
(+)-pinoresinol to (+)-lariciresinol. Chem Pharm Bull (Tokyo) 51, 508-515.

28. Mroczyńska M \& Libudzisz Z (2010) Beta-glucuronidase and beta-glucosidase activity of Lactobacillus and Enterococcus isolated from human feces. Pol J Microbiol 59, 265-269.

29. Costabile A, Klinder A, Fava F, et al. (2008) Whole-grain wheat breakfast cereal has a prebiotic effect on the human gut microbiota: a double-blind, placebo-controlled, crossover study. Br J Nutr 99, 110-120.

30. Kilkkinen A, Stumpf K, Pietinen P, et al. (2001) Determinants of serum enterolactone concentration. Am J Clin Nutr 73, 1094-1100.

31. Khalif IL, Quigley EM, Konovitch EA, et al. (2005) Alterations in the colonic flora and intestinal permeability and evidence of immune activation in chronic constipation. Dig Liver Dis 37, 838-849.

32. Nettleton JA, Greany KA, Thomas W, et al. (2004) Plasma phytoestrogens are not altered by probiotic consumption in postmenopausal women with and without a history of breast cancer. J Nutr 134, 1998-2003.

33. Kajander K, Krogius-kurikka L, Rinttilä T, et al. (2007) Effects of multispecies probiotic supplementation on intestinal microbiota in irritable bowel syndrome. Aliment Pharmacol Ther 26, 463-473.

34. Stumpf K, Pietinen P, Puska P, et al. (2000) Changes in serum enterolactone, genistein, and daidzein in a dietary intervention study in Finland. Cancer Epidemiol Biomarkers Prev 9, 1369-1372.

35. Jacobs DR, Pereira MA, Stumpf K, et al. (2002) Whole grain food intake elevates serum enterolactone. Br J Nutr 88, 111-116.

36. Nova E, Viadel B, Wärnberg J, et al. (2011) Beneficial effects of a synbiotic supplement on self-perceived gastrointestinal well-being and immunoinflammatory status of healthy adults. J Med Food 14, 79-85.

37. Marschan E, Kuitunen M, Kukkonen K, et al. (2008) Probiotics in infancy induce protective immune profiles that are characteristic for chronic low-grade inflammation. Clin Exp Allergy 38, 611-618.

38. Kajander K, Myllyluoma E, Rajilić-Stojanović $\mathrm{M}$, et al. (2008) Clinical trial: multispecies probiotic supplementation alleviates the symptoms of irritable bowel syndrome and stabilizes intestinal microbiota. Aliment Pharmacol Ther 27, 48-57. 